\title{
Routine checking of all manuscripts for plagiarism and duplicate publications
}

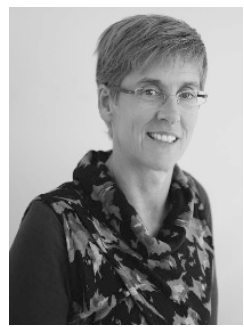

\author{
LA Harvey, MW Post, JD Steeves, MS Alexander and A Krassioukov \\ Spinal Cord Editorial Office, UK \\ E-mail: spinalcord@iscos.org.uk
}

Spinal Cord is now routinely checking all manuscripts for plagiarism and duplicate publications. ${ }^{1}$ This policy has also been adopted by Spinal Cord's affiliated journal: Spinal Cord Series and Cases. The checking is done by running all submitted manuscripts through automated software (CrossRef) that looks for phrases and text that perfectly match another source. The software then displays the matching text from the submitted manuscript side-by-side with the text from the second source for comparison. It also provides a score indicating the percentage of text in the manuscript that is taken from other sources. Nearly all manuscripts get a score of at least $5 \%$. This invariably reflects innocuous duplication including; text detailing authors' institutions, standard phrases or technical procedures. However, scores over $20 \%$ are investigated and scores over $35 \%$ are generally reflective of a serious problem.

Since the beginning of 2017 when routine checks on manuscripts were introduced, the Editorial Office of Spinal Cord has dealt with 8 manuscripts that had unacceptable levels of plagiarism (scores greater than 45\%) and another 4 manuscripts in which at least $30 \%$ of the text was taken from another source. Most of these submissions were rejected with no option for resubmission and a warning was issued about further actions the journal might take if there is a repeat offense, including formal notification to the authors' institutions and relevant funding agencies. The COPE discussion paper on plagiarism was used for guidance. $^{2}$

The most serious examples of plagiarism involved large sections of text being taken from other sources ad verbatim. This is particularly worrying if the text contains ideas and concepts which the authors are implying are their own. In these sorts of cases it is not sufficient to merely put a reference at the end of a section of text. This does not convey to the reader the critical point, namely that the text belongs to another author. Text like this must be placed in quotation marks with a reference including the page number from which the text was taken. Less serious examples of plagiarism include strings of sentences each taken from a different source.

Duplicate publications are also detected with the software screening because invariably authors self-plagiarise when submitting a second manuscript on the same topic. While self-plagiarism of short passages is sometimes inevitable, submitting papers for publication where the results have already been published previously is not acceptable unless there is a formal arrangement between publishers for co-publication of an important finding or policy statement. In this case, it is imperative that the second publication acknowledges the first publication. An overlapping second publication which has different results, but uses the same study cohort or dataset may be acceptable if key principles are followed, namely:

(i) All prior related publications and submissions are declared in the paper and the covering letter to the Editor at the time of submission with a clear statement about how the current publication differs from the others. These simple practices allow the Editor, reviewers and readers to value the merits of the paper in the light of related publications which may have preceded it.

(ii) Authors are upfront about the initial goals and hypotheses of their studies. Primary and secondary outcomes, along with any planned subgroup analyses, need to be articulated at the time of trial registration and/or within a study's protocol. Any analyses that are not planned before data collection commences should be considered exploratory and interpreted cautiously. In this way, nebulous or coincidental findings are not given the status of a primary finding in secondary publications; a practice that can mislead readers. ${ }^{3-5}$

Spinal Cord and Spinal Cord Series and Cases will continue to take plagiarism and unscrupulous duplicate publications seriously. Our firm stance on this issue is important to maintain our readers' confidence in the quality of the papers and the integrity of authors who submit their findings to our two journals.

1 Springer Nature. Plagarism and Fabrication. Accessed on 10th January 2017. http://www.nature.com/authors/policies/plagiarism.html.

2 Elizabeth Wager on behalf of COPE Council. How should editors respond to plagiarism? COPE Discussion document. Accessed on 10th January 2017. http://publicationethics.org/files/u7141/Discussion\%20document 0.pdf.

3 Ebrahim S, Montoya L, Kamal EI Din M, Sohani ZN, Agarwal A, Bance S et al. Randomized trials are frequently fragmented in multiple secondary publications. J Clin Epidemiol 2016; 79: 130-139.

4 Burke JF, Sussman JB, Kent DM, Hayward RA. Three simple rules to ensure reasonably credible subgroup analyses. BMJ $2015 ; 351$ : h5651.

5 Schulz KF, Grimes DA. Multiplicity in randomised trials II: Subgroup and interim analyses. Lancet 2005; 365: 1657-1661. 